\title{
HIGH RESOLUTION POLSAR IMAGE CLASSIFICATION BASED ON GENETIC ALGORITHM AND SUPPORT VECTOR MACHINE
}

\author{
P. X. Li ${ }^{\text {a, }}$, W. D. Sun ${ }^{\text {a }}$, J. Yang ${ }^{\text {a }}$, L. Shi ${ }^{\text {a }}$, F. K. Lang ${ }^{\text {a }}$, W. Jiang ${ }^{\text {a }}$ \\ ${ }^{a}$ The State Key Laboratory of Information Engineering in Surveying, Mapping and Remote Sensing of Wuhan \\ University, 430079, No.129 Luoyu Road, Wuhan, P. R. China - \\ hell.men@163.com
}

KEY WORDS: PolSAR, Classification, Feature Selection, GA, SVM

\begin{abstract}
:
This paper focuses on backscattering mechanisms selection and supervised classification works for CETC38-X PolSAR image. Thanks to the high radar resolution, many classes of man-made objects are visible in the images. So, land-use classification becomes a more meanful application using PolSAR image, but it involves the selection of classifiers and backscattering mechanisms. In this paper we apply SVM as the classifier and GA as the features selection method. Finally, after we find the best parameters and the suitable polarimetric information, the overall accuracy is up to $97.49 \%$. The result shows SVM is an effective algorithm compared to Wishart and BP classifiers.
\end{abstract}

\section{INTRODUCTION}

Thanks to the high radar resolution, now many classes of man-made objects, as well as vegetation and ground, are visible in PolSAR images so that PolSAR image classification is more significant. But unfortunately, previous works have shown us the problems of SAR image processing are far from being solved by a gain in resolution. Obviously, the high resolution helps discriminate small objects, but it creates new problems (Tison, 2004). So there requires a high robustness classifier. In the previous research, SVM (Support Vector Machine) is a good algorithm for classification and regression which is based on SRM (Structural Risk Minimization). In the recent literatures, it has been used for many fields, for instance, matching of SAR images and optical images (Hui, 2004), SAR image target recognition (Xue, 2005).

As we know, the 4-D coherency $\mathrm{T}_{4}$ matrix and the covariance $\mathrm{C}_{4}$ matrix are proposed in order to describe distributed targets, which can reduce to 3-D matrices for the reciprocity constrains. From $\mathrm{T}_{3}$ or $\mathrm{C}_{3}$, we can extract a lot of backscattering mechanism information based on polarimetric target decompositions. Intuitively, we try to obtain more helpful features to improve the classification accuracy, but the complicated relationships among them are always harmful. And thus we can first select the most useful backscattering features before classification. As a classical heuristic algorithm, GA (Genetic Algorithm) is routinely used to generate solutions to optimization and search problems (GoldBerg, 1989). In this paper, we simply utilize this algorithm to select features to enhance the subsequence classification accuracy.

\section{METHODS}

\subsection{Support Vector Machine}

In machine learning, SVM, also named support vector nerworks (Cortes, 1995), are supervised learning models with associated learning algorithms that analyze data and recognize patterns, used for classification and regression analysis.

For simplicity, let us first consider a supervised binary classification problem. Let us assume that the training set consists of $N$ vectors $x_{i} \in \mathfrak{R}^{d}(i=1,2, \cdots, N)$ from the d-dimensional feature space $X$. To each vector $x_{i}$, we associate a target $y_{i} \in\{-1,+1\}$. The linear SVM classification approach consists of looking for a separation between the two classes in $X$ by means of an optimal hyperplane that maximizes the separating margin. In the nonlinear case, which is the most commonly used as data are often linearly nonseparable, they are first mapped with a kernel method in a higher dimensional feature space $\Phi(X) \in \mathfrak{R}^{d^{\prime}}\left(d^{\prime}>d\right)$. The membership decision rule is based on the function $\operatorname{sign}[f(x)]$, where $f(x)$ represents the discriminant function associated with the hyperplane in the transformed space and is defined as

$$
f(x)=w^{*} \cdot \Phi(x)+b^{*}
$$

The optimal hyperplane defined by the weight vector $w^{*} \in \mathfrak{R}^{d^{\prime}}$ and the bias $b^{*} \in \mathfrak{R}$ is the one that minimizes a cost function

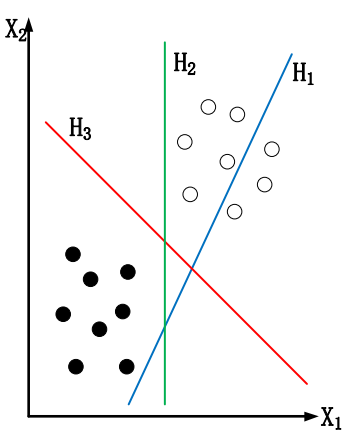

(a)

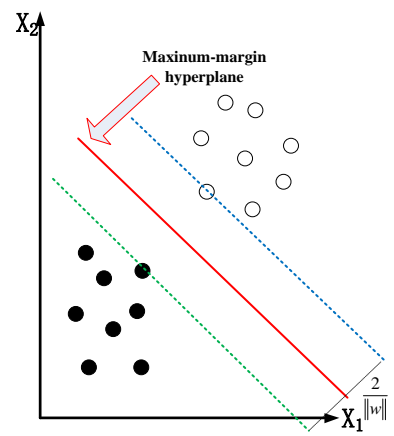

(b)

* E-mail address: pxli@whu.edu.cn 


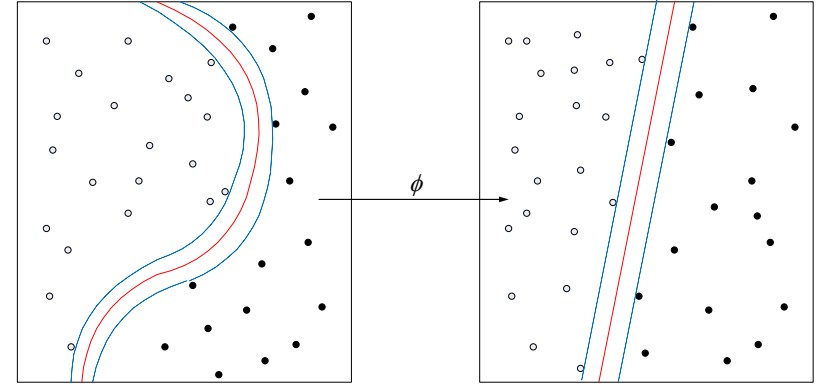

(c)

Figure 1. Discriminant hyperplane, (a) $\mathrm{H}_{1}$ does not separate the classes, $\mathrm{H}_{2}$ does, but only with a small margin, $\mathrm{H}_{3}$ separates them with the maximum margin. (b) Maxinum-margin hyperplane and margins for an SVM trained with samples from two classes, samples on the margin are called the support vectors. (c) Kernel machine for nonlinear classification.

that expresses a combination of two criteria,namely: 1) margin maximization and 2) error minimization. It is expressed as

$$
\Psi(w, \xi)=\frac{1}{2}\|w\|^{2}+C \sum_{i=1}^{N} \xi_{i}
$$

This cost function minimization is subject to the constrains

$$
\begin{aligned}
& y_{i}\left(w \cdot \Phi\left(x_{i}\right)+b\right) \geq 1-\xi_{i}, i=1,2, \cdots, N \\
& \xi_{i} \geq 0, i=1,2, \cdots, N
\end{aligned}
$$

where $\xi_{i}$ are the so-called slack variables introduced to account for nonseparable data. The contant $C$ represents a regularization parameter that allows to control the shape of the discriminant function and, consequently, the decision boundary when data are nonseparable. The above optimization problem can be reformulated as

$$
\max _{\alpha} \sum_{i=1}^{N} \alpha_{i}-\frac{1}{2} \sum_{i, j=1}^{N} \alpha_{i} \alpha_{j} y_{i} y_{j} K\left(x_{i}, x_{j}\right)
$$

under the constraints

$$
\begin{aligned}
& \alpha_{i} \geq 0, i=1,2, \cdots, N \\
& \sum_{i=1}^{N} \alpha_{i} y_{i}=0
\end{aligned}
$$

where $\alpha=\left[\alpha_{1}, \alpha_{2}, \cdots, \alpha_{N}\right]$ is a vector of Lagrange multipliers. The final result is a discriminant function conveniently expressed as

$$
f(x)=\sum_{i \in S} \alpha_{i}^{*} y_{i} K\left(x_{i}, x\right)+b^{*}
$$

where $K(\cdot, \cdot)$ is a kernel function. The set $S$ is a subset of the indices $\{1,2, \cdots, N\}$ corresponding to the nonzero Lagrange multipliers $\alpha_{i}$, which define the so-called support vectors. The kernel function we use is the Gaussian function

$$
K\left(x_{i}, x\right)=\exp \left(-\gamma\left\|x_{i}-x\right\|^{2}\right)
$$

where $\gamma$ represents a parameter inversely proportional to the width of the Gaussian kernel.

The basic SVM takes a set of input data and predicts, for each given input, which of two possible classes forms the output, making it a binary classifier. To apply on multi-classification, different multi-classification strategies can be adopted. Here we adopt one-against-one voting strategy.

\subsection{Genetic Algorithm}

GA is a search heuristic that mimics the process of natural evolution in the computer science field of artificial intelligence. It finds applications in a large of fields, such as engineering, chemistry, bioinformatics.

There have three main operations: 1) selection operation selects better individuals to keep down based on their fitness, 2) crossover operation recombines individuals according to a certain probability, 3) mutation operation keep the diversity of population, but its possibility must not be set too large.

In a genetic algorithm, a population of individuals to an optimization problem is evolved towards better solutions. Each individual has a set of chromosomes which can be mutated and altered. At first, individuals were represented only in binary code, later other encodings appeared. In this paper, we use binary code, so 0 and 1 stand for the existence of a corresponding feature.

The evolution starts from a population of randomly generated individuals and later updates every generation, namely is an iterative process. In each generation, the fitness of all individuals is evaluated; the fitness is usually the value of the objective function in the optimization problem, but here is the overall accuracy. The more fit individuals are more possible to preserve to next generation, and according to a certain probability, individuals are also probable to be recombined and randomly mutated, so finally there form a new generation. Until either the maximum number of generations or a satisfactory fitness level is achieved, the evolution stops. The best individual in the last generation is just what we want.

\section{EXPERIMENT}

\subsection{Experimental Data}

In this paper, there is a Paddyland data set. The CETC38-X Paddyland data set is acquired by the X-band Dual-antenna PolInSAR system in Linshui City of Hainan Province. The spatial resolution is about 0.5 meter in range and azimuth 
direction. The original image size is $2048 \times 2048$ pixels and the $5 \times 5$ pixels multi-look process is given to reduce the speckle noise, in Fig.2 (a); in Fig.2 (b), there are total five classes, respectively corresponding to paddy in different growth stages.

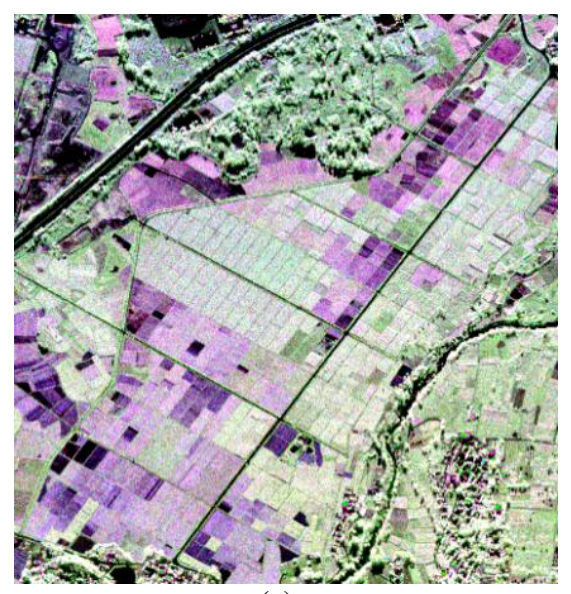

(a)

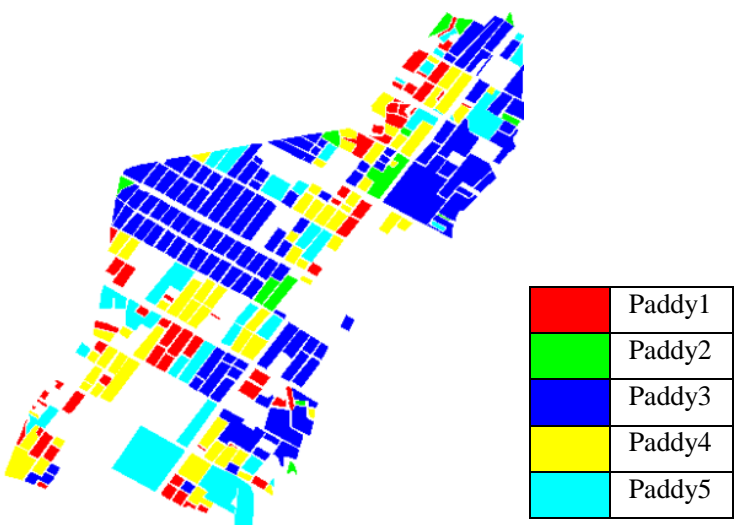

(b)

Figure 2. PolSAR image of Paddyland in Linshui City, (a) Pauli based on PolSAR image: red for $|\mathrm{HH}-\mathrm{VV}|$, green for $2|\mathrm{HV}|$, and blue for $|\mathrm{HH}+\mathrm{VV}|$. (b) Ground truth region of total five classes.

\subsection{Cross Validation}

Here SVM is used to achieve multi-classification. 30 samples of each class are randomly got as a training set. Applying SVM and Gaussian function, there are two parameters need to set: Gauss kernel $\gamma$ and penalty factor $C$. Setting them different values, the classification accuracy probably changes dramatically, which shows in Fig.3 (a)-(c). We use the Cross Validation (CV) algorithm to obtain the best $\gamma$ and $C$. The final experiment results show the best $\gamma$ and $C$ are 0.03125 and 445.7219, and the corresponding accuracy is $93.17 \%$ in Fig.3 (d).

\subsection{Features Selection}

After finding $\gamma$ and $C$, the next issue is multi-backscattering mechanisms selection. We utilize GA to select backscattering features. Coded binary form makes each gene in chromosomes stands for the existence of a feature. The overall classification accuracy is treated as fitness. The number of individuals and evolution generations are 30 and 100. In Fig.3 (e) and (f), it shows that the suitable features improve the classification accuracy to $97.49 \%$. TABLE 4 lists the features which we use in the beginning and which we select later.

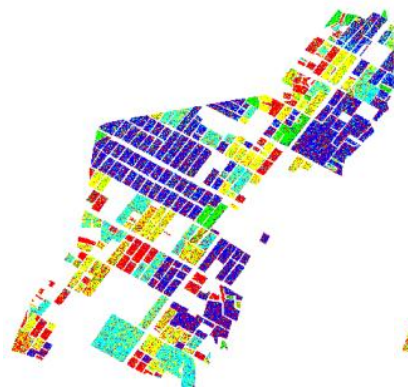

(a)

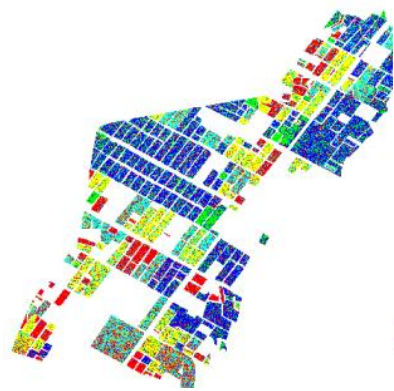

(c)

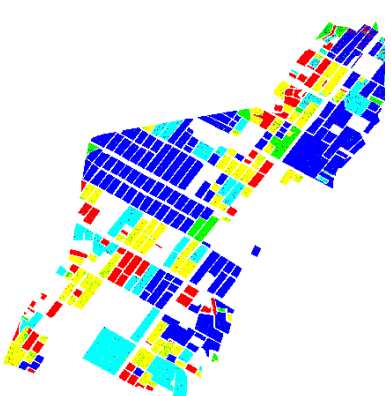

(e)

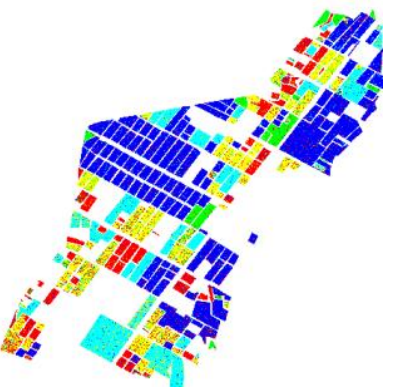

(b)

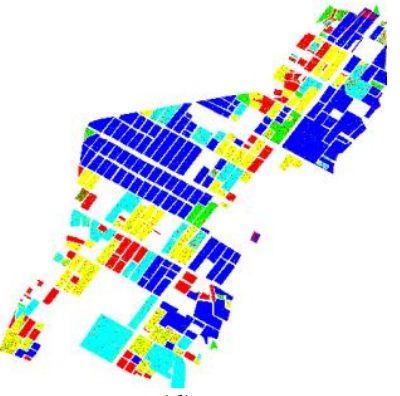

(d)

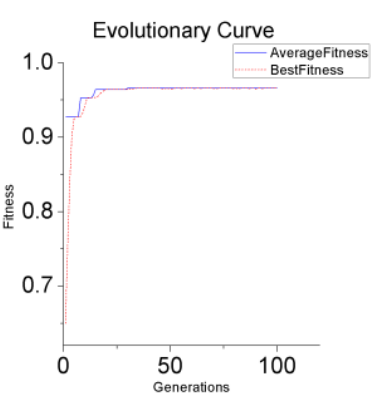

(f)

Figure 3. The influences of different combinations of $\gamma$ and $C$, (a) $\gamma=1$ and $C=10$, accuracy is $65.33 \%$. (b) $\gamma=0.1$ and $C$ $=300$, accuracy is $87.50 \%$. (c) $\gamma=0.5$ and $C=30$, accuracy is

$60.02 \%$. (d) $\gamma=0.03125$ and $C=445.7219$, accuracy is

$93.17 \%$. (e) Select suitable features to classify paddy, accuracy is $97.49 \%$. (f) GA race evolutionary curve.

\begin{tabular}{|c|c|c|c|}
\hline Features & Description & beginning & selection \\
\hline$\left|C_{11}\right|$ & $\begin{array}{l}\text { Modulus in } 1^{\text {st }} \\
\text { row and } 1^{\text {nd }} \\
\text { column in } C_{3}\end{array}$ & $\sqrt{ }$ & $\sqrt{ }$ \\
\hline$\left|C_{22}\right|$ & $\begin{array}{l}\text { Modulus in } 2^{\text {st }} \\
\text { row and } 2^{\text {nd }} \\
\text { column in } C_{3}\end{array}$ & $\sqrt{ }$ & $\sqrt{ }$ \\
\hline$\left|C_{33}\right|$ & $\begin{array}{l}\text { Modulus in } 3^{\text {st }} \\
\text { row and } 3^{\text {nd }} \\
\text { column in } C_{3}\end{array}$ & $\sqrt{ }$ & $\sqrt{ }$ \\
\hline$\left|C_{12}\right|$ & $\begin{array}{l}\text { Modulus in } 1^{\text {st }} \\
\text { row and } 2^{\text {nd }} \\
\text { column in } C_{3}\end{array}$ & $\sqrt{ }$ & $\sqrt{ }$ \\
\hline$\left|C_{13}\right|$ & $\begin{array}{l}\text { Modulus in } 1^{\text {st }} \\
\text { row and } 3^{\text {nd }} \\
\text { column in } C_{3}\end{array}$ & $\sqrt{ }$ & $\sqrt{ }$ \\
\hline$\left|C_{23}\right|$ & $\begin{array}{l}\text { Modulus in } 2^{\text {st }} \\
\text { row and } 3^{\text {nd }} \\
\text { column in } C_{3}\end{array}$ & $\sqrt{ }$ & / \\
\hline$\varphi_{12}$ & $\begin{array}{c}\text { Phase in } 1^{\text {st }} \text { row } \\
\text { and } 2^{\text {nd }} \text { column in } \\
C_{3}\end{array}$ & $\sqrt{ }$ & / \\
\hline
\end{tabular}




\begin{tabular}{|c|c|c|c|}
\hline$\varphi_{13}$ & $\begin{array}{c}\text { Phase in } 1^{\text {st }} \text { row } \\
\text { and } 3^{\text {nd }} \text { column in } \\
C_{3}\end{array}$ & $\sqrt{ }$ & / \\
\hline$\varphi_{23}$ & $\begin{array}{c}\text { Phase in } 2^{\text {st }} \text { row } \\
\text { and } 3^{\text {nd }} \text { column in } \\
C_{3}\end{array}$ & $\sqrt{ }$ & / \\
\hline Span & $\begin{array}{c}\text { Total power } \\
\left(=\mathrm{C}_{11}+\mathrm{C}_{22}+\mathrm{C}_{33}\right)\end{array}$ & $\sqrt{ }$ & $\sqrt{ }$ \\
\hline$H$ & $\begin{array}{c}\text { Polarimetric } \\
\text { entropy }\end{array}$ & $\sqrt{ }$ & $\sqrt{ }$ \\
\hline$A$ & $\begin{array}{c}\text { Polarimetric } \\
\text { anisotropy }\end{array}$ & $\sqrt{ }$ & / \\
\hline$\alpha$ & $\begin{array}{c}\text { Scattering } \\
\text { alpha angle }\end{array}$ & $\sqrt{ }$ & $\sqrt{ }$ \\
\hline$k_{D}$ & $\begin{array}{c}\text { Diplane } \\
\text { component }\end{array}$ & $\sqrt{ }$ & $\sqrt{ }$ \\
\hline$k_{H}$ & $\begin{array}{c}\text { Helix } \\
\text { component }\end{array}$ & $\sqrt{ }$ & / \\
\hline$k_{S}$ & $\begin{array}{c}\text { Sphere } \\
\text { Spre }\end{array}$ & $\sqrt{ }$ & $\sqrt{ }$ \\
\hline
\end{tabular}

TABLE 4. Feature Selection $(|\cdot|$ stands for Modulus; $\varphi$ stands for Phase; $H, A, \alpha$ are from $H, A, \alpha$ decomposition; $k_{D}, k_{H}, k_{S}$ are based on Krogager decomposition. )

\subsection{Contrast Results}

In this section, we compare SVM classifier with another two classifiers: Wishart and BPNN (back-propagation neural network), the classification results are in Fig.5 and TABLE 6.

In the previous research, literature (Lee, 2009) derived the complex Wishart measurement to classify PolSAR images. But the Wishart measurement does not work well in very high resolution SAR images. The supervised Wishart classification result is obtained in Fig.4 (c) and the overall classification accuracy is $74.85 \%$. Obviously, the Wishart classifier utilizes the covariance matrix and coherence matrix directly, but the physical decomposition results are omitted. On the other hand, the results display that the BP classifier also performances not very well in this data set. Among the three, only the overall accuracy from SVM classifier is good enough.

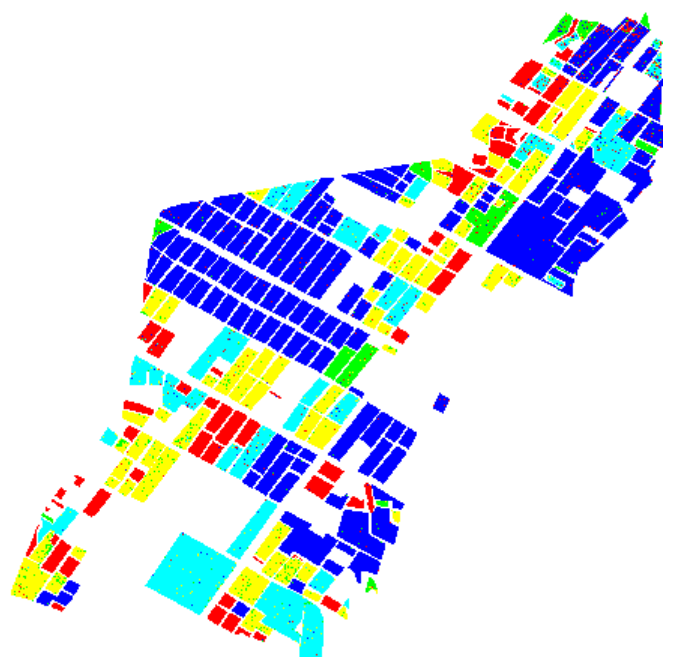

(a)

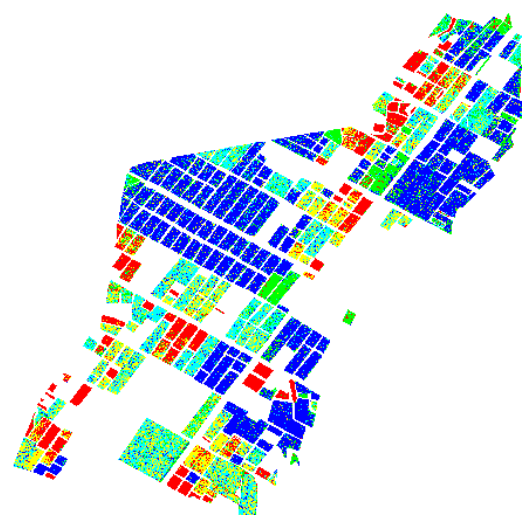

(b)

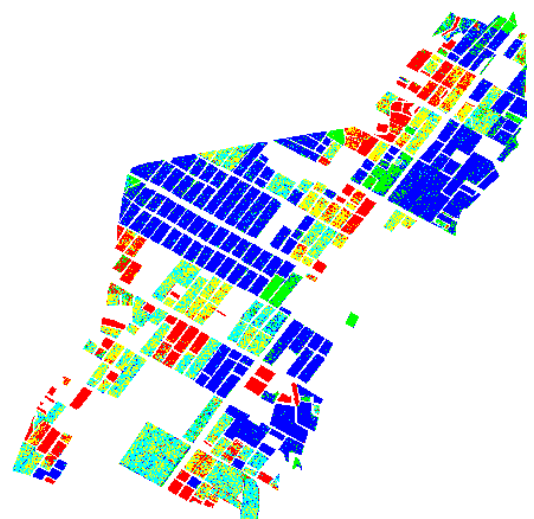

(c)

Figure 5. Contrast of three classifiers, (a) SVM classifier, accuracy is $97.49 \%$, kappa is 0.9641 . (b) BPNN classifier, accuracy is $70.12 \%$, kappa is 0.5887 . (c) Wishart classifier, accuracy is $74.85 \%$.

\begin{tabular}{|c|r|r|r|r|r|}
\hline Class & $\begin{array}{c}\text { Paddyla } \\
\text { nd 1 }\end{array}$ & \multicolumn{1}{c|}{$\begin{array}{c}\text { Paddyla } \\
\text { nd 2 }\end{array}$} & \multicolumn{1}{|c|}{$\begin{array}{c}\text { Paddyla } \\
\text { nd 3 }\end{array}$} & $\begin{array}{c}\text { Paddyla } \\
\text { nd 4 }\end{array}$ & \multicolumn{1}{c|}{$\begin{array}{c}\text { Paddyla } \\
\text { nd 5 }\end{array}$} \\
\hline $\begin{array}{c}\text { Paddyla } \\
\text { nd 1 }\end{array}$ & 98.67 & 1.09 & 0.10 & 0 & 0.14 \\
\hline $\begin{array}{c}\text { Paddyla } \\
\text { nd 2 }\end{array}$ & 1.55 & 93.87 & 0.40 & 4.18 & 0 \\
\hline $\begin{array}{c}\text { Paddyla } \\
\text { nd 3 }\end{array}$ & 1.05 & 0.42 & 98.43 & 0.01 & 0.08 \\
\hline $\begin{array}{c}\text { Paddyla } \\
\text { nd 4 }\end{array}$ & 0.40 & 2.16 & 0.01 & 97.28 & 0.15 \\
\hline $\begin{array}{c}\text { Paddyla } \\
\text { nd 5 }\end{array}$ & 1.92 & 0.20 & 0.70 & 1.65 & 95.53 \\
\hline
\end{tabular}

(a)

\begin{tabular}{|c|r|r|r|r|r|}
\hline Class & $\begin{array}{c}\text { Paddyla } \\
\text { nd 1 }\end{array}$ & \multicolumn{1}{|c|}{$\begin{array}{c}\text { Paddyla } \\
\text { nd 2 }\end{array}$} & $\begin{array}{c}\text { Paddyla } \\
\text { nd 3 }\end{array}$ & $\begin{array}{c}\text { Paddyla } \\
\text { nd 4 }\end{array}$ & $\begin{array}{c}\text { Paddyla } \\
\text { nd 5 }\end{array}$ \\
\hline $\begin{array}{c}\text { Paddyla } \\
\text { nd 1 }\end{array}$ & 90.93 & 2.65 & 0.08 & 5.15 & 1.19 \\
\hline $\begin{array}{c}\text { Paddyla } \\
\text { nd 2 }\end{array}$ & 2.76 & 77.76 & 8.11 & 1.58 & 9.80 \\
\hline $\begin{array}{c}\text { Paddyla } \\
\text { nd 3 }\end{array}$ & 0.37 & 8.29 & 80.11 & 3.85 & 7.38 \\
\hline $\begin{array}{c}\text { Paddyla } \\
\text { nd 4 }\end{array}$ & 13.88 & 5.27 & 4.43 & 48.25 & 28.17 \\
\hline $\begin{array}{c}\text { Paddyla } \\
\text { nd 5 }\end{array}$ & 1.77 & 11.69 & 7.35 & 23.55 & 55.65 \\
\hline
\end{tabular}

(b)

TABLE 6. Confusion matrix of SVM and BPNN, (a) Confusion matrix of SVM. (b) Confusion matrix of BPNN.

\section{CONCLUSIONS}

Thanks to the development of SAR sensors and imaging 
technology, we can observe many man-made objects in PolSAR images in recent years, and this brings PolSAR image classification tasks. Though the coherency $\mathrm{T}_{3}$ matrix and the covariance $\mathrm{C}_{3}$ matrix include a large number of polarimetric information, the blend information is useless to analysis targets precisely. So we need to extract different kinds of information based on polarimetric target decompositions. But how to effectively make use of these features, it is still an unresolved problem.

This paper introduces GA and SVM to high resolution PolSAR image classification. Firstly, we expound the theory of SVM and GA. Then, we verify the effectiveness of this method with the contrast of three classifiers using the CETC38-X Paddyland data set: the Wishart classifier directly use the $\mathrm{C}_{3}$ or $\mathrm{T}_{3}$ matrix, but can not use additional polarimetric information, and thus its overall accuracy is only $74.85 \%$; contrary to Wishart, the BPNN classifier can apply additional information, but theoretically it is probable to fall into local minimum, so its accuracy is not good yet; finally, the SVM classifier gets the best result, as a result of its SRM theory and the use of additional information, the accuracy is up to $97.49 \%$. Experimentally, it is obvious that SVM is a rather effective algorithm to classify high resolution PolSAR images. In future, we will focus on improving the classification capability via integration algorithm.

\section{REFERENCES}

Cortes, C., Vapnik, V., 1995. Support-Vector Networks. Machine Learning, 20, pp. 273-297.

Goldberg, D., 1989. Genetic Algorithms in Search, Optimization, and Machine Learning. Addison-Wesley Professional, New Jersey, pp. 1-21.

Hui, C., Zheng, S., Yu, Q., Tian, J., Liu, J., 2004. Matching of SAR images and optical images based on edge feature extracted via SVM. IEEE Signal Processing, 2, pp. 930-933.

Lee, J., Pottier, E., 2009. Polarimetric Radar Imaging. Chemical Rubber Company, Florida, pp. 265-274.

Tison, C., Nicolas, J., Tupin, F., 2004. A new statistical model for markovian classification of urban areas in high-resolution SAR images. IEEE Transactions on geoscience and remote sensing, 42(10), pp. 2046-2057.

Xue, X., Zeng, Q., Zhao, R., 2005. A new method of SAR image target recognition based on SVM. IEEE Geoscience and Remote Sensing Symposium, 7, pp. 4718-4721.

\section{ACKNOWLEDGEMENTS}

This work was supported by the National Natural Science Foundation under Grant 60890074 and in part by the National High-Technology R\&D Program (863 Program) under Grant 2011AA120404.

The experimental data used in this paper was provided by China Electronics Technology Group Corporation No.38 Research Institute (CETC38). 